\title{
Performance of the spin-dependent Krieger-Li-Iafrate approximation in jellium slabs
}

\author{
C. M. Horowitz \\ Instituto de Investigaciones Fisicoquímicas Teóricas y Aplicadas (INIFTA), UNLP, CCT La Plata-CONICET, \\ Diagonal 113 y 64, La Plata, Argentina \\ S. Rigamonti \\ Humboldt-Universität zu Berlin, Institut für Physik and IRIS Adlershof, 12489 Berlin, Germany \\ C. R. Proetto \\ Centro Atómico Bariloche and Instituto Balseiro, C.P. 8400, San Carlos de Bariloche, Río Negro, Argentina
}

(Received 8 February 2019; revised manuscript received 8 May 2019; published 6 June 2019)

\begin{abstract}
The possible spin-polarized states of metallic jellium slabs have been studied by means of a Krieger-LiIafrate (KLI) approximation for the exchange potential. The stability of the different magnetic states has been determined by using a fixed-spin-moment method. Notably, in the search for solutions with different magnetizations, the KLI approximation leads to the sudden filling of electronic states for either majority or minority spin channels, and also to hysteresis effects, as the system crosses these points of discontinuity. These effects are completely missed by the local spin-density approximation (LSDA), which exhibits instead a continuous behavior. Close to the density corresponding to the Bloch instability of the homogeneous 3D electron gas, different exotic magnetic configurations appear, some of them featuring a spontaneous breaking of inversion symmetry around the slab center. As compared to the LSDA, the spin-dependent KLI approximation offers a moderate increase of the stability of a slab antiferromagnetic state.
\end{abstract}

DOI: 10.1103/PhysRevB.99.235111

\section{INTRODUCTION}

Most of the codes aimed at the ab initio calculation of electronic properties of solids contain, in one way or another, some ingredients coming from density-functional theory (DFT) [1-3]. Its widespread use is mainly based on the reasonable balance between accuracy and computational cost, offered by most of the standard approximations [local density approximation (LDA), generalized gradient approximation (GGA), meta-GGA, etc.]. While approximations to the exchange-correlation energy kernel are very common and in some cases unavoidable (correlation) in DFT, since its very proposal the search for more accurate approximations has never stopped [4]. Within this context, the development of the optimized effective potential (OEP) method [5] within the Kohn-Sham (KS) formulation of DFT has contributed in giving precise recipes about how the exchange and correlation potentials should be obtained from the corresponding exchange and correlation energy functionals [6,7]. When restricted to exchange alone, omitting correlation, the OEP method has also been denoted as exact-exchange (EXX) or $x$-only OEP ( $x$-OEP).

In a previous paper [8], we developed a general DFTOEP formal scheme for quasi-two-dimensional electron gas (Q2DEG) systems, either isolated (closed) or in contact with a particle bath (open). A typical example of a Q2DEG closed system is the one of the present paper: a metallic slab with a jellium approximation for the discrete positive ions [9-12]. On the other side, in DFT-OEP calculations of the electronic structure of $n$-doped semiconductor quantum wells, the same are usually considered as open Q2DEGs [13,14]. In fact, in Ref. [8] we have shown that both possible representations (open or closed) of a given Q2DEG system are equivalent, the choice of one or the other being essentially a question of convenience. The formalism is flexible enough as to include the possibility of having spontaneously spin-polarized inhomogeneous ground states. The aim of this paper is to provide numerical examples of this spin-dependent formalism, measuring its performance for the exchange potential, and for the specific case of a metallic slab, in the jellium approximation for the discrete positive ions.

Regarding the application of the DFT-EXX method to "real" insulator and semiconductor slabs, the same has been mainly developed in the last years by Engel [15-19]. In these works, the electronic structure of thin slabs of graphene and $\mathrm{Si}(111)$ has been studied in detail, both numerically through EXX (in the KLI approximation) plane-wave pseudopotential calculations, and also analytically. Among the main results, he has proven that the exchange potential behaves like $-e^{2} / z$ in the asymptotic (vacuum) region also for real slabs [20], and that the EXX calculations are feasible for these systems, at least within the widely used and most of the time accurate KLI-approximation to the full OEP exchange potential [21].

The possible spin-polarized states of thin metallic slabs have been studied in the past. Okazaki and Teraoka (Ref. [22]) obtained the ground states for this system in the low-density limit, using the local-spin-density approximation (LSDA) for the exchange-correlation kernel. Several states, such as nonmagnetic $(\mathrm{N})$, fully polarized ferromagnetic (FPF), partially polarized ferromagnetic (PPF), and antiferromagnetic (AF) 
were found, depending on the slab width $d$. The same problem has been discussed by Sen in Ref. [23], that studied the electronic and magnetic structures in (100) films of K and Cs, having thicknesses of one to seven layers. The calculations for these "real" metallic slabs were inside the plane-wave projector augmented wave formalism of DFT, using both LSDA and a GGA. Only a six-layer Cs film was found to have a ferromagnetic state, degenerate with a paramagnetic state within the accuracy of the calculations. To extract the behavior of the spin-dependent exchange potential that we are proposing here in the cleanest possible way, correlation effects will be omitted in all of our EXX(KLI) calculations. For this reason, we also also an extended set of reference $x$-only LSDA calculations, useful for comparison with the EXX(KLI) ones. We have verified that LSDA results including correlation effects (not shown) compare well with those of Ref. [22].

The rest of the paper is organized as follows: in Sec. II, the essentials of the spin-dependent KLI exchange potential are given; Sec. III is devoted to introduce the fixed-spin-moment (FSM) method and to provide the reference set of $x$-only LSDA results; Sec. IV is devoted to the $x$-only KLI results, including a ground-state determination. The conclusions are given in Sec. V.

\section{KRIEGER-LI-IAFRATE APPROXIMATION FOR JELLIUM SLABS}

Our calculations are restricted to a jellium-slab model, where the discrete character of the positive ions inside the metal is replaced by a uniform distribution of positive charge (the jellium) $[24,25]$. If not stated otherwise, Hartree atomic units are used throughout this work [26]. The positive jellium density is defined as

$$
n^{+}(z)=n_{0} \theta\left(\frac{d}{2}-|z|\right),
$$

where $d$ is the width of the slab with edges at $z=-d / 2$ and $z=d / 2$ and [27] $n_{0}=3 /\left(4 \pi r_{s}^{3}\right) ; \theta(x)$ represents the Heaviside step function: $\theta(x)=1$ if $x>0$ and $\theta(x)=0$ if $x<0$. The positive jellium generates an attractive potential that confines the movement of electrons in the $z$ direction, while they are free to move along the $x-y$ plane. Defined this way, the slab has global (but not local) charge neutrality. From a more general perspective, this jellium-slab model represents also an example of a Q2DEG [28,29]. In the Q2DEG, the electrons are also confined in one spatial direction by a confinement potential, while they are free to move in the perpendicular direction. In the limit of very wide slabs $\left(d / \lambda_{F} \gg 1\right)$, it also becomes a model of a metallic surface $[24,25]$.

If translational invariance in the $x-y$ plane is assumed, and the confinement coordinate is $z$, the single-particle $\mathrm{KS}$ orbitals can be written as a product of a plane wave along $\rho_{\|}=(x, y)$ and a so-called subband orbital in $z$,

$$
\psi_{\mathbf{k} i \sigma}(\mathbf{r})=\frac{e^{i \mathbf{k} \cdot \boldsymbol{\rho}_{\|}}}{\sqrt{A}} \xi_{i \sigma}(z),
$$

where $\mathbf{k}=\left(k_{x}, k_{y}\right)$ is the in-plane wave vector, $A$ is the area of the Q2DEG, $i$ is the subband index $(=1,2,3, \ldots)$, and $\sigma$ the spin index (up or down). The full factorization between the solution in the $x-y$ plane and the $z$ direction is only valid for a local potential [30]; since the OEP method lies within the framework of the KS implementation of DFT, the factorization implies no lack of generality, owing to the locality of the KS potential. On the other side, the assumption of translational invariance in the $x-y$ plane does imply some lack of generality. According to the results in Ref. [31], however, the assumption is well justified for the relatively high 2D densities typically found in Q2DEGs. The energies $E_{\mathbf{k} i \sigma}=k^{2} / 2+\varepsilon_{i \sigma}$ of the single-particle KS orbitals in Eq. (2) are the sum of a continuous free-electron spectrum $k^{2} / 2$ corresponding to the in-plane movement, and a discrete subband spectrum $\varepsilon_{i \sigma}$ related to the confined motion in the jellium self-generated quantum well. The subband orbitals $\xi_{i \sigma}(z)$ are obtained from the self-consistent solution of the KS equation along the confinement coordinate,

$$
\left[-\frac{1}{2} \frac{\partial^{2}}{\partial z^{2}}+V_{s \sigma}(z)\right] \xi_{i \sigma}(z)=\varepsilon_{i \sigma} \xi_{i \sigma}(z),
$$

where the KS potential $V_{s \sigma}(z)=V_{e}(z)+V_{\mathrm{H}}(z)+V_{x c \sigma}(z)$, with $V_{e}(z)$ being the external potential, $V_{\mathrm{H}}(z)$ the Hartree potential, and $V_{x c \sigma}(z)$ is the exchange-correlation $(x c)$ potential. The latter may be split in the sum of its exchange and correlation contributions: $V_{x c \sigma}(z)=V_{x \sigma}(z)+V_{c \sigma}(z)$. Since the Hamiltonian in Eq. (3) is real, the subband wave functions can be taken to be real without loss of generality [32]. The main quantity in spin-dependent DFT is the spin-resolved 3D electronic density, that for our slab geometry is given by

$$
n_{\sigma}(z)=\sum_{i} \theta_{i \sigma} n_{i \sigma} \xi_{i \sigma}(z)^{2},
$$

where the occupation factors $n_{i \sigma}$ are

$$
n_{i \sigma}=\frac{\mu-\varepsilon_{i \sigma}}{2 \pi},
$$

with $\theta_{i \sigma} \equiv \theta\left(n_{i \sigma}\right)$ and $\mu$ denotes the chemical potential.

The total number of electrons per unit area $n$ (hereafter the areal density) is given by the integral over all $z$ of Eq. (4),

$$
n=\sum_{i \sigma} \theta_{i \sigma} n_{i \sigma}=\frac{N_{s}}{2 \pi} \mu-\sum_{i \sigma} \frac{\theta_{i \sigma}}{2 \pi} \varepsilon_{i \sigma},
$$

where $N_{s}=\sum_{i \sigma} \theta_{i \sigma}$ is the total number of occupied subbands. By imposing the condition $n_{0} d=n$, the slab global charge neutrality is satisfied, and the chemical potential $\mu$ determined. Let us note that, as given above, $n^{+}(z), n_{\sigma}(z), n_{i \sigma}$, and $n$ are dimensionless densities; to recover the corresponding dimensions, the right-hand side (rhs) of the corresponding equations should be multiplied by $a_{0}^{-3}, a_{0}^{-3}, a_{0}^{-2}$, and $a_{0}^{-2}$, respectively. Typically, the areal density is about $10^{14}-10^{15}$ electrons $/ \mathrm{cm}^{2}$ for the case of metallic jellium slabs.

Additional convenient variables for spin-polarized systems are the spin-density polarization $m(z)$, which relates to the spin resolved density through

$$
m(z)=n_{\uparrow}(z)-n_{\downarrow}(z),
$$

and the global relative spin polarization $\eta$, given by

$$
\eta=\frac{1}{n} \int_{-\infty}^{\infty} m(z) d z .
$$


An important parameter for the present paper is the slab magnetic moment per unit area $M$, defined by

$$
M=\eta n_{0} d,
$$

in units of $\mu_{B}$, the Bohr magneton. Besides constants, and for fixed slab size $(d), \eta$ and $M$ are equivalent physical quantities.

In a previous paper, we have developed a general DFTOEP formal scheme valid both for Q2DEG's either isolated (closed) or in contact with a particle bath (open) [8]. In the present case of an isolated jellium-slab, the physical situation corresponds to a closed system with fixed areal density $n$. This imposes the following constraint on the allowed density perturbations:

$$
\sum_{\sigma=\downarrow, \uparrow} \int_{-\infty}^{\infty} \delta^{n} n_{\sigma}(z) d z=0
$$

where $\delta^{n} n_{\sigma}(z)$ denotes a spin-density variation that leaves $n$ unchanged. That is, in a closed system, the change in the total number of electrons, as expressed by the integral of Eq. (10), must be exactly zero. Using this constraint and the fact that the set of variables $\left\{V_{s \sigma}(z), n\right\}$ and $\left\{V_{s \sigma}(z)+K, n\right\}$ determine exactly the same density $n_{\sigma}(z)$, where $K$ is a spin-independent constant, it is possible to obtain the complete set of equations that must satisfy the DFT-OEP scheme [8].

In this context, the Krieger-Li-Iafrate (KLI) [33] approximation for the exchange potential $V_{x \sigma}(z)$ can be written as [8]

$$
V_{x \sigma}(z) \simeq V_{x \sigma}^{\mathrm{KLI}}(z)=\sum_{i} \frac{\theta_{i \sigma} n_{i \sigma} \xi_{i \sigma}(z)^{2}}{n_{\sigma}(z)}\left[u_{x i \sigma}(z)+\overline{\Delta V}_{x i \sigma}\right],
$$

where $u_{x i \sigma}(z)$ is the orbital potential given by [34]

$$
u_{x i \sigma}(z)=\frac{1}{2 A n_{i \sigma}} \frac{1}{\xi_{i \sigma}(z)} \frac{\delta E_{x}}{\delta \xi_{i \sigma}(z)},
$$

and

$$
\overline{\Delta V}_{x i \sigma}=\int_{-\infty}^{\infty} \xi_{i \sigma}(z)^{2}\left[V_{x \sigma}^{\mathrm{KLI}}(z)-u_{x i \sigma}(z)\right] d z .
$$

In Eq. (12), $E_{x}=E_{x \uparrow}+E_{x \downarrow}$ is the OEP exchange energy functional, defined below.

In addition, an important constraint has to be fulfilled [8],

$$
\bar{C}_{x \sigma}=\bar{C}_{x \underline{\sigma}} \equiv \bar{C}_{x}
$$

where the overline indicates $\bar{C}_{x \sigma}=\sum_{i} C_{x i \sigma} / N_{s \sigma}$, $\underline{\sigma}$ indicates the spin projection opposite to $\sigma$, and the scalars $C_{x i \sigma}$ are defined by [35]

$$
\begin{aligned}
C_{x i \sigma} & =\theta_{i \sigma} \int_{-\infty}^{\infty} \xi_{i \sigma}(z)^{2} V_{x \sigma}^{\mathrm{KLI}}(z) d z-\frac{1}{A} \frac{\partial E_{x}}{\partial n_{i \sigma}} \\
& =\theta_{i \sigma} \bar{V}_{x \sigma}^{i}-\frac{1}{A} \frac{\partial E_{x}}{\partial n_{i \sigma}} .
\end{aligned}
$$

The constraint of Eq. (14) admits an elegant interpretation in a representation in which the independent variables $n_{\uparrow}(z)$ and $n_{\downarrow}(z)$ are replaced by $n(z)=\sum_{\sigma} n_{\sigma}(z)$ and $m(z)$. In this case, the change in total energy around the equilibrium densities contains the term $\mu_{B}\left(\bar{C}_{x \uparrow}-\bar{C}_{x \downarrow}\right) \delta M / 2$. Thus, Eq. (14) expresses the stability of the closed interacting system with

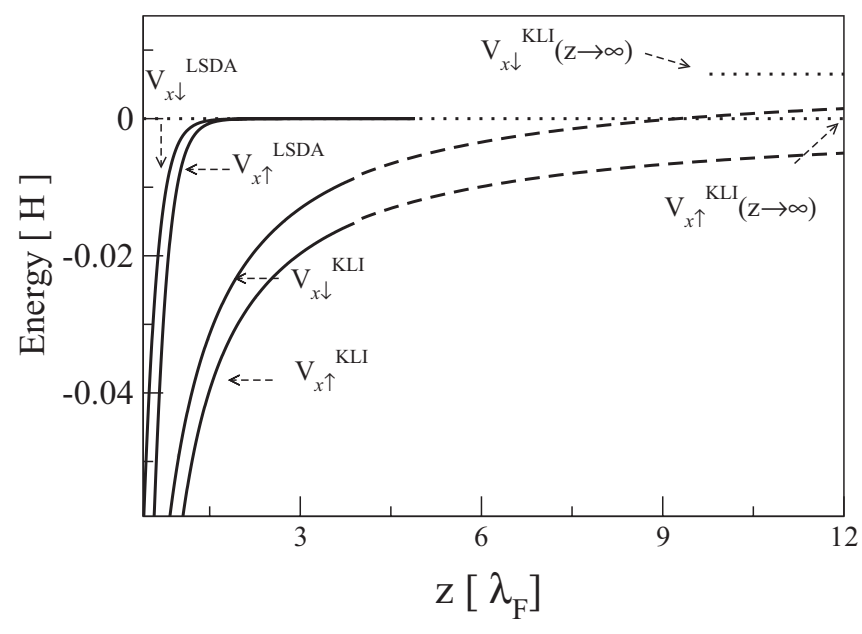

FIG. 1. Asymptotic behavior of the spin-dependent exchange potential in the $x$-KLI approximation, for $r_{s}=5.0$ and $d / \lambda_{F}=0.8$. Full curves represent $V_{x \sigma}^{\mathrm{KLI}}(z)$ and $V_{x \sigma}^{\mathrm{LSDA}}(z)$, with $\sigma=\uparrow(\sigma=\downarrow)$ for the majority (minority) spin component. The dashed curves are asymptotic approximations for the many-subband $x$-KLI exchange potential (see text for more details), and the two arrows on the right signal the two different asymptotic limits for the $\uparrow$ and $\downarrow$ KLI exchange potentials.

respect to changes in the total magnetization. A thorough derivation of this can be found in Appendix B of Ref. [8].

Since, in the closed system, $V_{x \sigma}^{\mathrm{KLI}}(z)$ is defined up to a floating constant, one can choose (either for $\sigma=\uparrow$ or $\sigma=\downarrow$, but not for both) that $V_{x \sigma}^{\mathrm{KLI}}(z \rightarrow \infty) \rightarrow 0$ by imposing the condition $\overline{\Delta V}_{x m_{\sigma} \sigma}=0$, that implies that $\bar{V}_{x m_{\sigma} \sigma}=\bar{u}_{x m_{\sigma} \sigma}$, where $m_{\sigma}$ is the index of the highest occupied subband [36]. On the other side, the other spin component of the exchange potential $V_{x \underline{\alpha}}^{\mathrm{KLI}}(z)$ is fully determined by the constraint in Eq. (14), and then has the asymptotic limit $V_{x \underline{\sigma}}^{\mathrm{KLI}}(z \rightarrow \infty) \rightarrow$ $\overline{\Delta V}_{x m_{\underline{\sigma}} \underline{\sigma}}=\bar{V}_{x m_{\underline{\alpha}} \underline{\sigma}}-\bar{u}_{x m_{\underline{\alpha}} \underline{\sigma}}$, which in general will be different from zero.

As an explicit example of this asymptotic behavior, we display in Fig. 1 the exchange potential $V_{x \sigma}^{\mathrm{KLI}}(z)$ for values of $z$ deep in the vacuum region for the case $r_{s}=5.0$ and $d / \lambda_{F}=0.8$. The asymptotic limit of the minority spin component (with just one occupied subband) is given by $V_{x \downarrow}^{\mathrm{KLI}}(z \rightarrow \infty) \rightarrow 0.0065 \mathrm{H}-e^{2} / z\left(1-8.932 a_{0} / z\right)$. On the other side, the asymptotic limit for the majority spin component (with two occupied subbands) is given by $-e^{2} / z(1-$ $9.045 a_{0} / z$ ), according to our choice of the floating constant. In addition, for comparison, Fig. 1 shows, for the same slab, the decay of the $V_{x \sigma}^{\mathrm{LSDA}}(z)$. From this comparison, the much slower decay of the $x$-KLI exchange potentials becomes evident, as compared with the $x$-LSDA. Another explicit example of this remarkable asymptotic behavior of the exchange potential is given in Fig. 3 of Ref. [8], within the framework of the full EXX approach.

The total energy per unit area, considering the slab geometry and neglecting correlation is given by

$$
E(d, \eta)=E_{\mathrm{el}}(d, \eta)+\sum_{\sigma}\left[E_{K \sigma}(d, \eta)+E_{x \sigma}(d, \eta)\right],
$$


where

$$
E_{K \sigma}(d, \eta)=\sum_{i}^{\text {occ }} n_{i \sigma}\left[2 \pi n_{i \sigma}-\int_{-\infty}^{\infty} \xi_{i \sigma}(z) \frac{\partial^{2} \xi_{i \sigma}(z)}{\partial z^{2}} d z\right]
$$

is the spin-dependent kinetic energy contribution,

$$
E_{\mathrm{el}}(d, \eta)=\frac{1}{2} \int_{-\infty}^{\infty} V_{\mathrm{H}}(z)\left[n(z)-n^{+}(z)\right] d z
$$

is the electrostatic energy due to all noncompensated positive and negative charge distributions in the slab, and

$$
\begin{aligned}
E_{x \sigma}(d, \eta)= & -\sum_{i, j} \theta_{i \sigma} \theta_{j \sigma} \int_{-\infty}^{\infty} \int_{-\infty}^{\infty} \xi_{i \sigma}(z) \xi_{j \sigma}(z) \xi_{i \sigma}\left(z^{\prime}\right) \xi_{j \sigma}\left(z^{\prime}\right) \\
& \times \frac{g\left(\sqrt{4 \pi} n_{i \sigma} \Delta z, \sqrt{4 \pi} n_{j \sigma} \Delta z\right)}{4 \pi(\Delta z)^{3}} d z d z^{\prime}
\end{aligned}
$$

is the spin-dependent exchange energy contribution. In the last equation, $\Delta z=\left|z-z^{\prime}\right|$, and

$$
g\left(s, s^{\prime}\right)=s s^{\prime} \int_{0}^{\infty} \frac{J_{1}(s t) J_{1}\left(s^{\prime} t\right)}{\sqrt{1+t^{2}}} \frac{d t}{t}
$$

is the "universal" function introduced by Kohn and Mattsson [37], with $J_{1}(x)$ being the first-order cylindrical Bessel function [38].

In the $x$-only LSDA, the exchange potential $V_{x \sigma}^{\mathrm{KLI}}(z)$ is replaced by $V_{x \sigma}^{\mathrm{LSDA}}(z)=-\left[12 n_{\sigma}(z) / \pi\right]^{1 / 3}$, while the exchange energy is expressed as

$$
E_{x \sigma}^{\mathrm{LSDA}}(d, \eta)=-\left(\frac{81}{32 \pi}\right)^{1 / 3} \int_{-\infty}^{\infty} n_{\sigma}(z)^{4 / 3} d z
$$

The corresponding total energy per unit area for the slab will be denoted as $E^{\mathrm{LSDA}}(d, \eta)$. Eqs. (19) and (20) are two different approximations for the slab exchange energy functional. The increased computational cost associated with the calculation of the exchange potential from the OEP expression stems from the fact that the latter is an implicit functional of the density, while $E_{x \sigma}^{\mathrm{LSDA}}(d, \eta)$ is an explicit functional of same. Note the nonphysical explicit exponential decay of $V_{x \sigma}^{\mathrm{LSDA}}(z)$ for $z$ taking values far in the vacuum region, dictated by the (physically correct) exponential asymptotic decay of $n_{\sigma}(z)$. This should be contrasted with the physically correct decay of $V_{x \sigma}^{\mathrm{KLI}}(z)$ discussed above. Both features are clearly seen in Fig. 1. Regarding the accuracy of the KLI approximation, all previous evidence in Q2DEG systems point to the fact that the KLI exchange potential is very close to the full EXX exchange potential. This has been checked explicitly for the same particular slab of Fig. 1 (see Fig. 2, top panel, in Ref. [8]). Besides, the KLI exchange potential becomes the exact one in the one-subband regime for each spin component $[8,9]$.

\section{FIXED-SPIN-MOMENT METHOD AND $x$-ONLY LOCAL SPIN DENSITY RESULTS}

To find the different magnetic states present in the metallic jellium slabs, we resort to the FSM method [39-41]. In the FSM method, the magnetic moment of the system $(M)$ is constrained to have a fixed value and thus a particular magnetic solution is forced. Using this constraint, it is possible

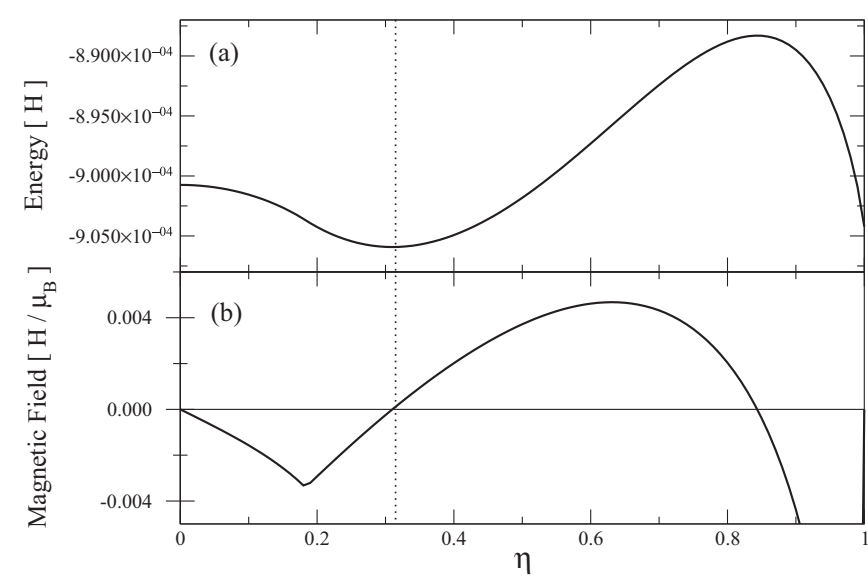

FIG. 2. $x$-LSDA total energy (top panel) and external magnetic field (lower panel) as a function of $\eta$, for $r_{s}=5$ and $d / \lambda_{F}=0.68$. The vertical line at $\eta \simeq 0.31$ signals the location of the stable PPF configuration.

to explore the energy landscape of the system $E(d, \eta)$ for different values of $M$ and to identify stable magnetic structures. Two approaches are used to implement this constrained search in the magnetic moment. In the first approach, the forced magnetic solution can be achieved through the application of an external magnetic field $\mathcal{H}$. The main drawback of this approach is that the function $M(\mathcal{H})$ usually is nonmonotonic and during the self-consistent process it is difficult to adjust the value of $\mathcal{H}$ to obtain the desired solution. In a different approach that is more practical, this constraint is accomplished by introducing two separate Fermi levels for each spin electronic state $[39,41]$. These Fermi levels are varied in the self-consistent calculation so as to get the desired magnetic solution.

As mentioned above, it is then possible to detect the different stable magnetic structures through the study of the function $E(M) \equiv E(d, \eta)$. For a fixed volume, stable solutions are presented by $M$ values corresponding to points where $\mathcal{H}=d E / d M=0$ and $d^{2} E / d M^{2}>0$ [39-41]. Therefore, there are two possible ways of determining the stable magnetic states: (i) as the points where the function $E(M)$ has minima, and (ii) as the points of the function $\mathcal{H}(M)$, where $\mathcal{H}=0$ and its derivative is positive.

Previous results in metallic jellium slabs, based on the spin DFT within the framework of the LSDA using the JanakMoruzzi-Williams exchange-correlation energy [42,43], determined the existence of several stable magnetic states [22]. Magnetic states with symmetrical spin densities (even with regard to the slab center) such as $\mathrm{N}(\eta=0), \operatorname{PPF}(0<\eta<1)$, and $\operatorname{FPF}(\eta=1)$ states, and a state that breaks that symmetry, the AF state, were reported [22]. In what follows, we will briefly describe the stable magnetic states obtained through the FSM method for metallic jellium slabs in the $x$-only local spin-density approximation ( $x$-LSDA), as they represent a good guide for the main study of this paper that is based on the $x$-only KLI approach ( $x$-KLI). As in Ref. [22], we will differentiate states with symmetrical spin densities regarding the slab center (symmetric states) or without this symmetry [nonsymmetric (NS) states]. 


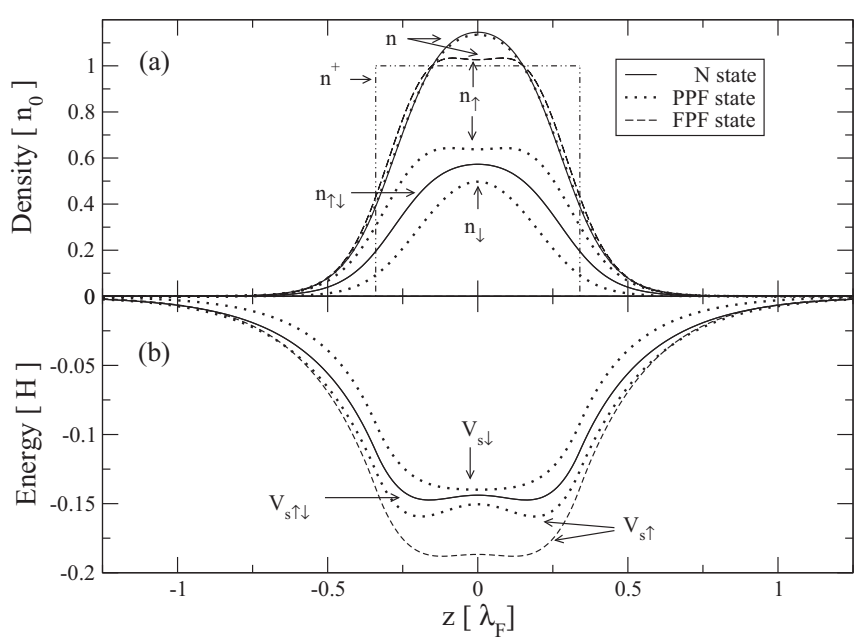

FIG. 3. $x$-LSDA spin-density profiles (top panel) and effective potentials (lower panel), for the configurations at $\eta=0(\mathrm{~N}), \eta \simeq$ 0.31 (PPF), and $\eta=1$ (FPF) in the previous figure.

As discussed above, to find efficiently magnetic states with a given value of $\eta$, we introduce two separate chemical potentials for each set of spin subbands. To obtain final states with symmetrical spin densities, we started the selfconsistent procedure with initial spin densities that fulfill this condition. In the same way, final states without that symmetry were searched with initial NS spin densities. Once the selfconsistent solution is obtained, we compute the total energy per unit area and the external magnetic field from Eq. (16), and through the relation $\mathcal{H}=\left(\mu^{\uparrow}-\mu^{\downarrow}\right) / 2$, respectively, where $\mu^{\uparrow(\downarrow)}$ is the chemical potential for a spin-up (-down) set of subbands. The units of $\mathcal{H}$ are $\left[\mathrm{H} / \mu_{B}\right]$.

Figure 2(a) shows the calculated total energy $E(d, \eta)$ as a function of $\eta$ obtained for symmetric states in the $x$-LSDA for a slab with $r_{s}=5.0$ and $d / \lambda_{F}=0.68$. Also, the external magnetic field $\mathcal{H}$ versus $\eta$, for the same case, is shown in Fig. 2(b). From both figures, two stable states can be recognized, the FPF state $(\eta=1)$ [44] and the PPF state with a value of $\eta \simeq 0.31$. The $\mathrm{N}$ state at $\eta=0$ is the paramagnetic one $\left(\eta^{*}(z) \equiv 0\right)$ and can only be obtained by requiring, in addition to the spin-densities being symmetrical, the condition of having both spin-densities matched. The spin-density profiles and the effective potentials for these N, FPF, and PPF states are shown in Figs. 3(a) and 3(b), respectively. The small "bump" that appears in some of the potentials around the slab center is a consequence of the occupation of the second subband slab state, whose wave-function $\xi_{2 \sigma}(z)$ has a node at $z=0$.

For symmetric states, the computed $E(d, \eta)$ and $\mathcal{H}(d, \eta)$ in metallic jellium slabs up to a slab width $d / \lambda_{F}=2$ show, in addition to the paramagnetic state, the appearance of FPF states in slabs with $r_{s} \gtrsim 4$ (for $r_{s} \lesssim 3$ the FPF state is only stable on the thinnest slabs), and the stability of PPF states in some widths of slabs for $r_{s} \gtrsim 4$. The case $r_{s}=5.0$ is the one with the greatest variety of PPF states. For this case, contour plots of $E(d, \eta)$ and $\mathcal{H}(d, \eta)$ are shown in Figs. 4 and 5, respectively. In Fig. 4, the stable states (energy minima) are indicated with black symbols, the energy maxima are indicated with red ones, and the absolute extremes are

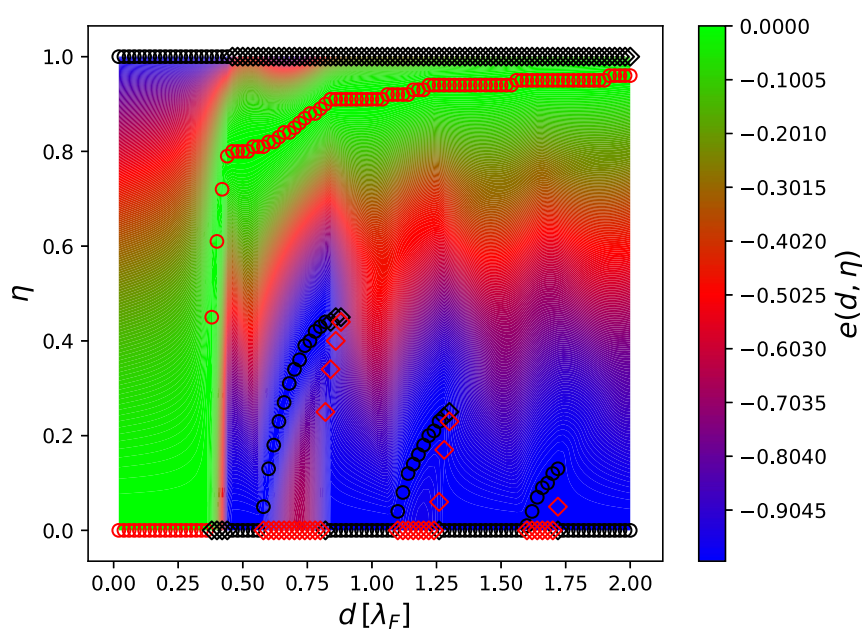

FIG. 4. $x$-LSDA rescaled total energy $e(d, \eta)=[E(d, \eta)-$ $\left.E_{\max }\right] /\left(E_{\max }-E_{\min }\right)$ (color scale) in the $\eta$ vs $d$ plane, for $r_{s}=5$. Minima are indicated with black symbols, maxima with red symbols. Absolute extremes, either minimum or maximum, are represented with circles of the corresponding color. Due to the rescaling, $e=0$ represents the maximum energy in the whole data set $\left(E_{\max }\right)$ while $e=-1$ the minimum $\left(E_{\min }\right)$.

represented with circles. In Fig. 5, black and red symbols indicate the points where the external magnetic field are null with $d \mathcal{H} / d \eta>0$ and $d \mathcal{H} / d \eta<0$, respectively. Both figures show the emergence of stable PPF states for slab widths within the following intervals $0.60 \lesssim d / \lambda_{F} \lesssim 0.85,1.10 \lesssim$ $d / \lambda_{F} \lesssim 1.30$, and $1.60 \lesssim d / \lambda_{F} \lesssim 1.74$. Also, this systematic study shows that, for $r_{s}=6.0$ and $d / \lambda_{F} \gtrsim 1.08$, there exist stable $\mathrm{N}$ states which are nonparamagnetic, i.e., $\eta=0$ but $\eta^{*}(z) \neq 0$. This $\mathrm{N}$ nonparamagnetic state is of lower energy than the paramagnetic one and its main characteristic is the

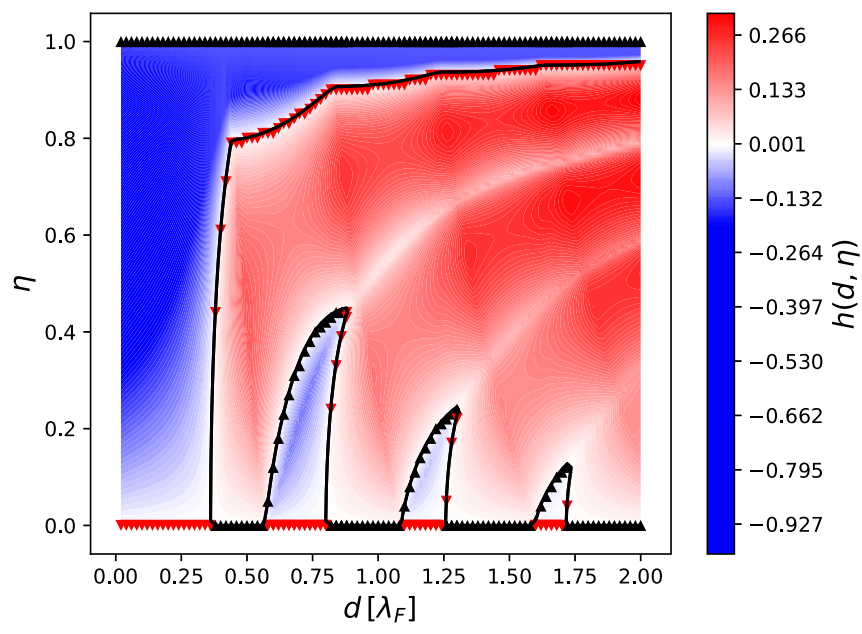

FIG. 5. $x$-LSDA rescaled external magnetic field $h(d, \eta)=$ $\mathcal{H}(d, \eta) /\left(\mathcal{H}_{\max }-\mathcal{H}_{\min }\right)$ (color scale) in the $\eta$ vs $d$ plane for $r_{s}=$ 5. Points where $\mathcal{H}(d, \eta)=0$ (white color in the color map), are denoted with black symbols for stable magnetic configurations $(d \mathcal{H}(d, \eta) / d \eta>0$ for $\eta<1)$ and with red symbols for unstable ones $(d \mathcal{H}(d, \eta) / d \eta<0) . \mathcal{H}_{\max }\left(\mathcal{H}_{\min }\right)$ is the maximum (minimum) field in the data set. 


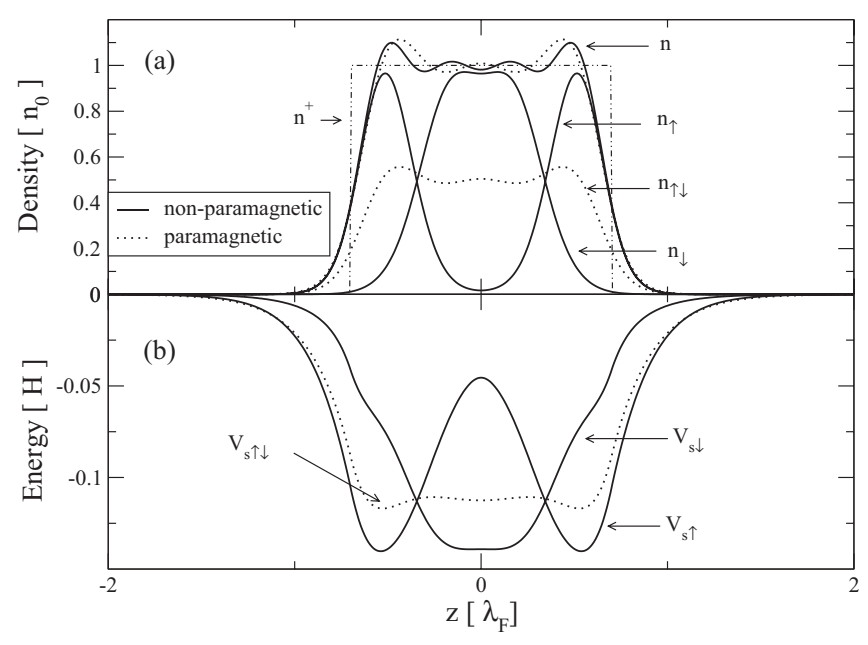

FIG. 6. $x$-LSDA spin-density profiles (top panel) and effective KS potentials (lower panel) for $r_{s}=6$ and $d / \lambda_{F}=1.40$. The dotted lines correspond to the $\mathrm{N}$ paramagnetic state with $m(z) \equiv 0$, the full lines to the $\mathrm{N}$ nonparamagnetic state with $m(z) \neq 0$.

presence of opposite spin-density polarization $[m(z)]$ between the center and the edges of the slab. To exemplify this, the spin-density profiles and the effective potentials of both $\mathrm{N}$ states (the paramagnetic and nonparamagnetic states) for the case of $r_{s}=6.0$ and $d / \lambda_{F}=1.40$ are shown in Figs. 6(a) and $6(\mathrm{~b})$, respectively.

From the range of considered densities, NS states are only observed in metallic jellium slabs with $r_{s}=5.00$ and $r_{s}=6.00$. There are NS states of two different types: states where $n_{\sigma}(z)=n_{\sigma}(-z)$ for which $\eta=0$ (AF state), and states with $\eta \neq 0$ (NS-PPF states). The systematic study of $E(d, \eta)$ and $\mathcal{H}(d, \eta)$ surfaces shows that the AF states are stable in slab widths within the interval $0.56 \lesssim d / \lambda_{F} \lesssim 0.64$ for $r_{s}=5$ and, in slab widths $0.52 \lesssim d / \lambda_{F}$ for $r_{s}=6$. In all cases, the AF state has lower energy than the paramagnetic $\mathrm{N}$ state. While the AF state implies necessarily some loss of kinetic energy by the electrons of a given spin being localized roughly on half of the slab width, at low enough densities this is more than compensated by the gain in exchange energy. Figure 7(a) shows the spin-density profiles of the AF state for the particular case of $r_{s}=6.0$ and $d / \lambda_{F}=1.40$. Also, the effective potentials for the same particular case are shown in Fig. 7(b). The NS-PPF states only appear in slab widths $0.88 \lesssim d / \lambda_{F}$ for $r_{s}=6$. This state has the peculiarity of presenting an electron density not symmetrical with respect to the center of the slab. Figure 8 shows the spin-density profiles of these NS-PPF states for the case of $r_{s}=6.0$ and $d / \lambda_{F}=1.70$. In this case, two stable states are observed, a state of higher energy where $\eta=0.20$ [shown in Fig. 8(a)] and a state with $\eta=0.63$ [shown in Fig. 8(b)]. The main feature here is that $n(z) \neq n(-z)$. While this is not easy to grasp directly from the densities, we also display the difference $\Delta n(z)=n(z)-n(-z)$, that vanishes for a symmetric state, but is different from zero for an asymmetric state. Note that the size for $\Delta n(z)$ is given by the right-vertical axis, whose scale is smaller by a factor of about $10^{-2}$ as compared with the left-vertical axis. In brief, the asymmetry exists, but is very small $\left(n_{0} \gg|\Delta n(z)|\right)$. It is important to clarify that this

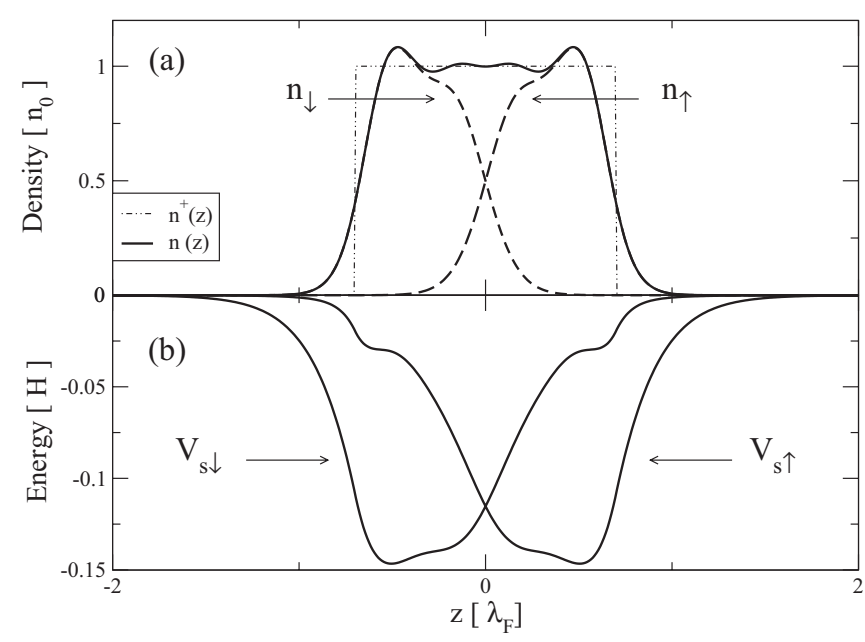

FIG. 7. $x$-LSDA spin-density profiles (top panel) and effective KS potentials (lower panel) for $r_{s}=6$ and $d / \lambda_{F}=1.40$, for the $\mathrm{AF}$ configuration. Note that since $n_{\sigma}(z)=n_{\underline{\sigma}}(-z)$, then $V_{s \sigma}(z)=$ $V_{s \underline{\sigma}}(-z)$. This is characterized as an $\mathrm{AF}$ state.

NS-PPF state is not a pathological one due to the absence of correlation. We have found that this same state appears in LSDA including correlation effects as in Ref. [22] in the low-density regime $\left(r_{s}=6.00\right)$ for $0.96 \lesssim d / \lambda_{F} \lesssim 1.04$ and $1.28 \lesssim d / \lambda_{F} \lesssim 1.32$

By comparing the total energy of all possible states, the ground state can be determined for each $r_{s}$ value and slab width. Figure 9 shows the magnetic configuration of the ground states in metallic jellium slabs up to slab widths $d / \lambda_{F}=2$. As expected, in the wider slabs the fundamental state is of the FPF symmetric type at low electronic densities $\left(r_{s}=6\right)$ and of the $\mathrm{N}$ symmetric paramagnetic type at higher electronic densities $\left(r_{s} \lesssim 4\right)$. For sufficiently narrow slabs, the ground state corresponds to an FPF symmetric state, even for high electronic densities. The case $r_{s}=5$ presents the greatest

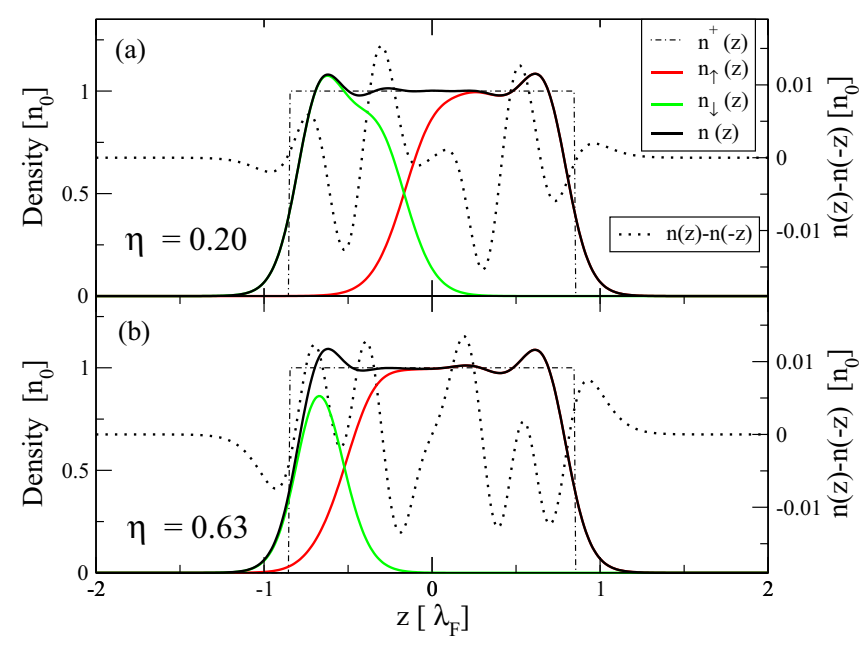

FIG. 8. $x$-LSDA spin-density profiles corresponding to two configurations with $\eta=0.20$ (top panel), and $\eta=0.63$ (lower panel) for $r_{s}=6$ and $d / \lambda_{F}=1.70$. Note that for these states, $n(z) \neq n(-z)$ (see text). Both cases are characterized as NS-PPF states. 


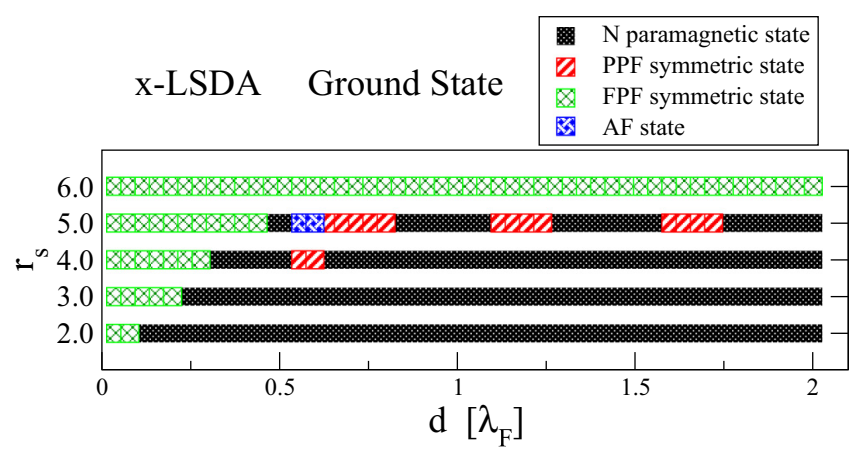

FIG. 9. Jellium slab ground-state configurations as resulting from $x$-only total energy LSDA calculations in the $r_{s}$ vs $d$ plane.

variety of fundamental states. In this case, it is observed that depending on the slab width, the ground state can be the FPF symmetric, N paramagnetic, PPF symmetric, or AF state. This is clearly related to the fact that for $r_{s} \simeq 5.45$ the homogeneous 3D electron gas has a paramagnetic-ferromagnetic instability first suggested by Bloch [45]. In our finite-size slab, as the density approaches this critical value, one should expect that the system be close to several possible stable states.

\section{IV. $x$-ONLY KLI RESULTS AND DISCUSSION}

The procedure used with the FSM method to find the stable magnetic states in the $x$-KLI approximation is slightly different from that used for the $x$-LSDA case (previous section).

On one side, it is well known that the $x$-KLI approach is an approximation to the full EXX approach, which is the method that seeks for that local potential that minimizes the total energy of the system when correlation is neglected and the exchange component of the total energy is given by the EXX energy functional [33]. For the case of metal slabs, this component is given by Eq. (19). But in $x$-KLI, the approximation is made at the level of the exchange potential, by appealing to a kind of "mean-field" argument [6,7]. As a consequence, there is no $x$-KLI energy functional from where the $x$-KLI potential may be obtained, and the stable states obtained in the $x$-KLI approach do not have to be states that minimize the total energy of the system [46]. As a result, a significant qualitative difference is expected in the $E(d, \eta)$ curve obtained for the $x$-KLI case with that obtained for the $x$-LSDA case. In the case of $x$-KLI, the total energy of the system at its minimum does not have to represent stable states. Therefore, unlike the case $x$-LSDA considered in the previous section, the stable magnetic states in the $x$-KLI approximation can only be recognized through the curve $\mathcal{H}(d, \eta)$, more precisely from the points where $\mathcal{H}=0$ and its derivative is positive. To illustrate the above, Fig. 10(a) shows the calculated total energy $E(d, \eta)$ [Eqs.(16)-(19)] as a function of $\eta$ obtained for symmetric states in the $x$-KLI approximation for the case $r_{s}=5.0$ and $d / \lambda_{F}=0.72$ in a range of $\eta$ values around a stable PPF state. Also, the external magnetic field $\mathcal{H}(d, \eta)$ versus $\eta$, for the same case, is shown in Fig. 10(b). As can be seen from the figure, the minimum energy is at $\eta \simeq 0.29$ and this value does not match the $\eta$ value of the stable state $\operatorname{PPF}(\eta \simeq 0.27)$.

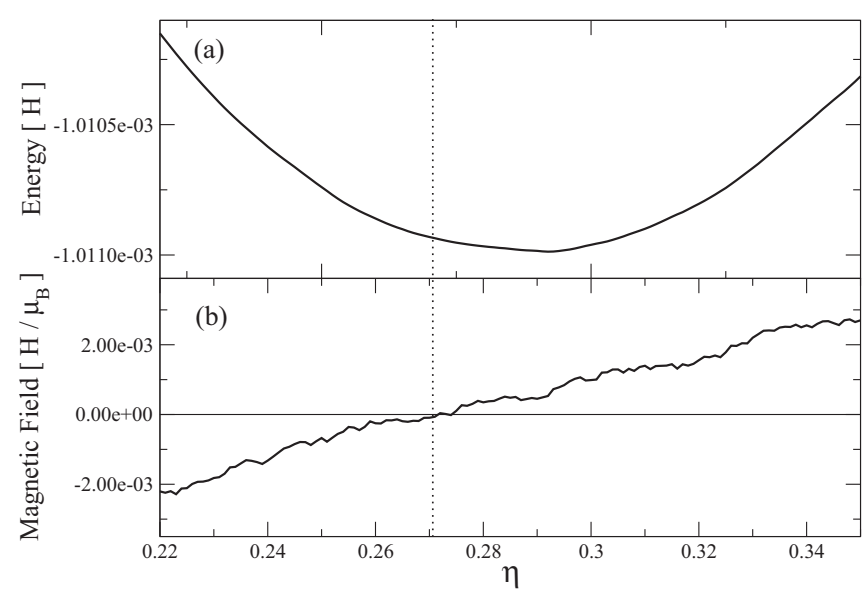

FIG. 10. $x$-KLI total energy $E(d, \eta)$ (top panel) and external magnetic field $\mathcal{H}(d, \eta)$ (lower panel) as a function of the global relative spin polarization $\eta$, for $r_{s}=5$ and $d / \lambda_{F}=0.72$. The vertical line at $\eta \simeq 0.27$ signals the stable PPF configuration, that differs from the point were $d E / d \eta=0(\eta \simeq 0.29)$.

In addition to this difference in the FSM procedure for determining stable states between the $x$-KLI and $x$-LSDA approaches, there are also qualitative differences in the $\mathcal{H}(d, \eta)$ and $E(d, \eta)$ curves between the two approaches. Figure 11(a) shows the external magnetic field $\mathcal{H}$ versus $\eta$ obtained for symmetric states in the $x$-KLI approximation for the specific case $r_{s}=2.0$ and $d / \lambda_{F}=0.30$. The evolution of the occupation factors for the same case is shown in Fig. 11(b). In both figures, the calculations are carried out with either increasing (black squares) and decreasing (red circles) $\eta$ values. On the one hand, it can be observed that, in a region close to $\eta=0.38$, the behavior of $\mathcal{H}(d, \eta)$ is different if the systematic study is performed by increasing or decreasing the values of $\eta$. As can be seen in Fig. 11(b), in this region the subband configuration of the system changes. More specifically, there is a small range of $\eta$ 's $(0.36 \lesssim \eta \lesssim 0.38)$ for which the occupancy of the second subband with spin up is either null (for increasing $\eta$ ) or finite (for decreasing $\eta$ ). This dependence

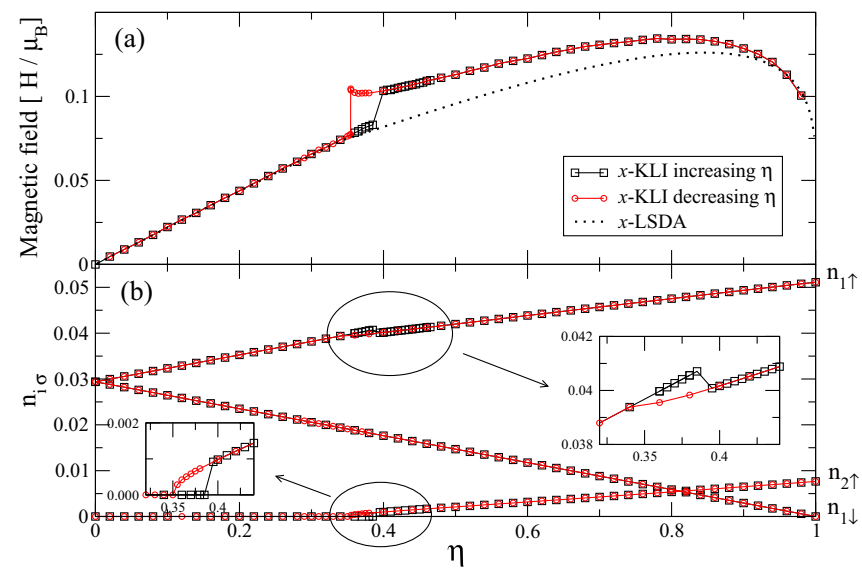

FIG. 11. $x$-KLI external magnetic field (top panel) and subband occupancy (lower panel) as a function of $\eta$, for $r_{s}=2$ and $d / \lambda_{F}=$ 0.30 . In the first case, $x$-only LSDA results are also displayed. 


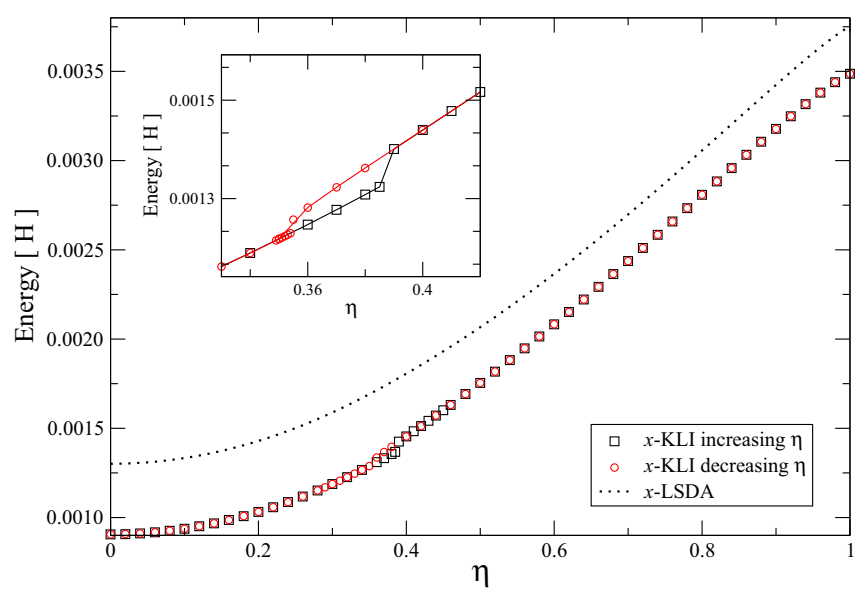

FIG. 12. Total energy $E(d, \eta)$ as a function of $\eta$, for $r_{s}=2.00$ and $d / \lambda_{F}=0.30$. Both $x$-only LSDA and KLI results are displayed. Inset: Enhanced view around the abrupt jump in the $x$-KLI solution.

of the subband configuration on the history also reflects in $\mathcal{H}(d, \eta)$, which displays a hysteresis behavior in the same range of $\eta$ 's. In the $x$-LSDA case, this does not happen because the subband configuration [and then also $\mathcal{H}(d, \eta)$ ] does not depend on its history in the whole range of $\eta$. On the other hand, besides the mentioned hysteresis, discontinuities in the behavior of the occupation factors and $\mathcal{H}(d, \eta)$ are observed in the same region for $x$-KLI. These discontinuities show another qualitative difference with respect to $x$-LSDA results, in which this fact is not observed. These discontinuities, associated with abrupt subband configuration changes, are also present in other computed quantities. Figure 12 shows how a discontinuity is also present in the total energy of the system, for the same case.

These two $x$-KLI characteristics shown for the case $r_{s}=2.0$ and $d / \lambda_{F}=0.30$, the hysteresis and the discontinuities, have been found in all the cases studied in regions where a change in the subband configuration of the system occurs. As this particular case, the branch with the lowest energy in the region of hysteresis corresponds to the configuration that occupies the smallest number of subbands. The size of the region of hysteresis, as well as the intensity of the discontinuities, are very dependent, however, on the value of $r_{s}$ and the width of the slab studied. More specifically, these effects are very noticeable in high-density regime $\left(r_{s}=2-3\right)$ and narrow slabs, while they tend to disappear in the opposite density regime and wide slabs.

\section{A. Magnetic structures in the $x$-KLI approximation}

\section{Symmetric states}

The thorough study of the $\mathcal{H}(d, \eta)$ values in metallic jellium slabs up to slab widths $d / \lambda_{F}=2$ and $r_{s}=2-6$ shows, in addition to the paramagnetic state in all cases, the appearance of FPF states in slabs with $r_{s} \gtrsim 4$. As in $x$-LSDA, for $r_{s} \lesssim 3$ the FPF state is only stable on the thinnest slabs. More explicitly, in $r_{s}=2$ and $r_{s}=3$, the FPF state is stable for $d / \lambda_{F} \lesssim 0.24$ and $d / \lambda_{F} \lesssim 0.68$, respectively. These stability regions are larger than in the $x$-LSDA case, which are $d / \lambda_{F} \lesssim 0.16$ for $r_{s}=2$ and $d / \lambda_{F} \lesssim 0.52$ for $r_{s}=3$. Since

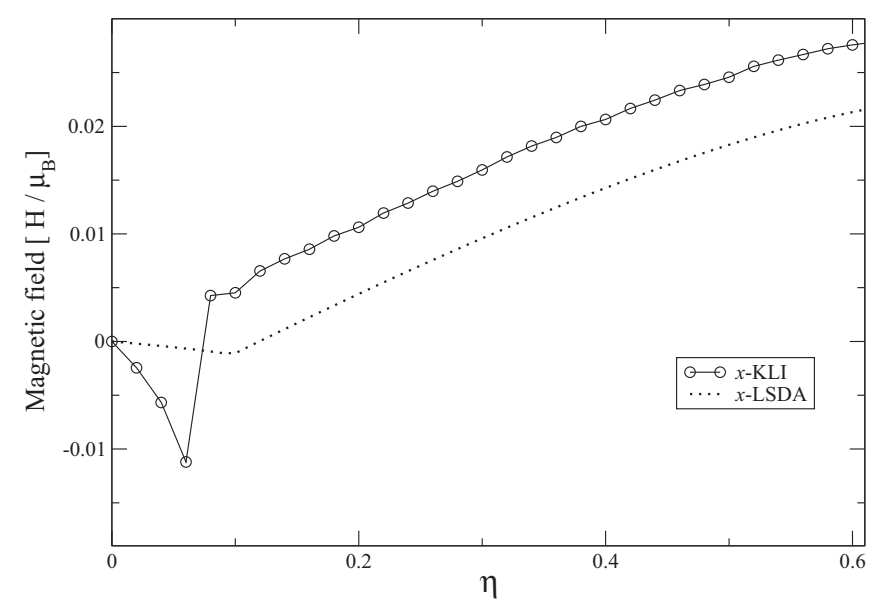

FIG. 13. External magnetic field $\mathcal{H}(d, \eta)$ as a function of $\eta$, for $r_{s}=4.00$ and $d / \lambda_{F}=0.60$. Both $x$-only LSDA and KLI results are displayed.

the FPF state is induced by the exchange interaction, one must conclude that the $x$-LSDA underestimates exchange effects, as compared with $x$-KLI.

Unlike $x$-LSDA, where in the region $0.56 \lesssim d / \lambda_{F} \lesssim 0.64$ for $r_{s}=4$ the PPF state is stable, in the case of $x$-KLI no stability of the PPF state is observed in the whole region studied for the case $r_{s}=4$. Figure 13 shows the external magnetic field $\mathcal{H}$ versus $\eta$, obtained for symmetric states in $x$-LSDA and $x$-KLI approximations for a slab width $d / \lambda_{F}=0.60$ and $r_{s}=4.0$. As can be seen from the figure, the discontinuity of $\mathcal{H}(d, \eta)$ caused by the change of system configuration (the second spin-down subband becomes emptied) is decisive for the absence of stability of the PPF phase in the case of $x$-KLI. The external magnetic field never becomes zero, unlike $x$-LSDA where it varies continuously from negative to positive values when increasing $\eta$. For values of $r_{s}=5$ and $r_{s}=6$, the PPF state presents stability in some slab widths. As in the case of $x$-LSDA, $r_{s}=5$ presents the greatest variety of PPF states for slab widths less than $2 \lambda_{F}$. The behavior of $\mathcal{H}(d, \eta)$ for the case $r_{s}=5$ shows the following intervals where the PPF state is stable: $0.68 \lesssim d / \lambda_{F} \lesssim 0.84,1.20 \lesssim$ $d / \lambda_{F} \lesssim 1.32$, and $1.72 \lesssim d / \lambda_{F} \lesssim 1.80$. These intervals are a bit smaller than the stability intervals of $x$-LSDA (see Sec. III). As for $r_{s}=4$, the discontinuities present in the curve $\mathcal{H}(d, \eta)$ for $x$-KLI are crucial for the nonoccurrence of stability in some slab widths and therefore the decrease of these stability intervals for $r_{s}=5$ and $r_{s}=6$.

As in $x$-LSDA, the $x$-KLI results show the existence of an $\mathrm{N}$ state $(\eta=0)$ other than the paramagnetic one for $r_{s}=6.0$ and $1.0 \lesssim d / \lambda_{F}$. Again, as in $x$-LSDA this $\mathrm{N}$ nonparamagnetic state is of lower energy than the paramagnetic one and its main characteristic is to present opposite spin-density polarization $[m(z)]$ between the center and the edges of the slab. The spin-density profiles of the $\mathrm{N}$ nonparamagnetic state for the case of $r_{s}=6.0$ and $d / \lambda_{F}=1.40$ in the $x$-KLI and $x$-LSDA approximations are shown in Fig. 14. In general, both approximations show very similar spin-density profiles for the $\mathrm{N}$ nonparamagnetic state, as can be seen in the particular case shown in Fig. 14. 


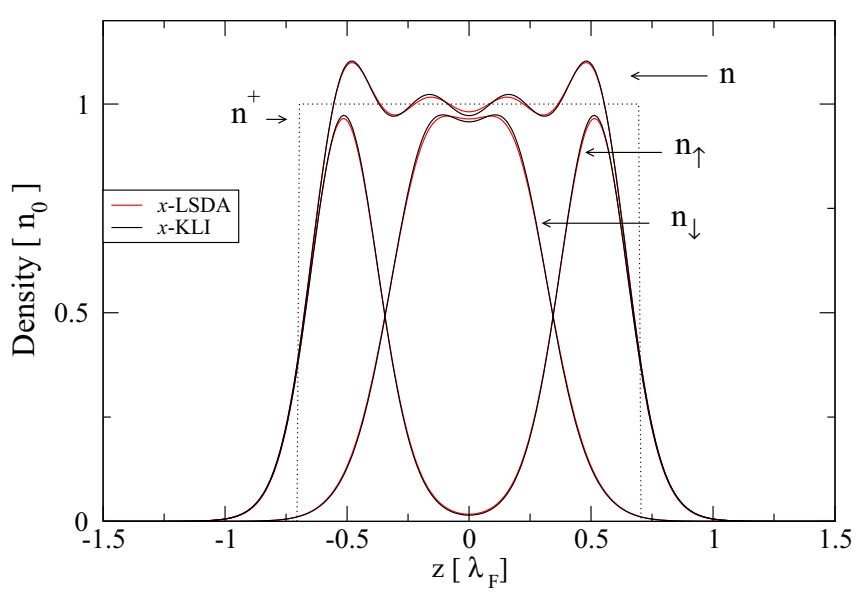

FIG. 14. $x$-KLI and $x$-LSDA spin-density profiles for $r_{s}=6.0$ and $d / \lambda_{F}=1.40$. The $x$-only LSDA and KLI profiles are almost on top of each other. In both approximations, this is a $\mathrm{N}$ nonparamagnetic stable state.

\section{Nonsymmetric states}

For the whole range of densities considered, NS states are only observed in metallic jellium slabs with $r_{s}=5.0$ and $r_{s}=$ 6.0 , as in $x$-LSDA.

The AF state, that is characterized by $n_{\sigma}(z)=n_{\sigma}(-z)$ and $\eta=0$, is stable in slab widths within the intervals $0.56 \lesssim$ $d / \lambda_{F} \lesssim 0.72,1.08 \lesssim d / \lambda_{F} \lesssim 1.16$ and $1.56 \lesssim d / \lambda_{F} \lesssim 1.72$ for $r_{s}=5$. Unlike what happens in $x$-LSDA, in this case the regions of stability of the AF phase extend to wider slab regions, containing a greater number of occupied subbands. For $x$-LSDA, this phase is observed in a range in which the configuration of the system consists of an occupied subband in each component of spin, while in $x$-KLI the first interval in which this phase appears corresponds to the same configuration, but the following intervals correspond to a configuration of two and three occupied subbands in each spin component. As examples, Figs. 15(a) and 15(b) show the spin-density

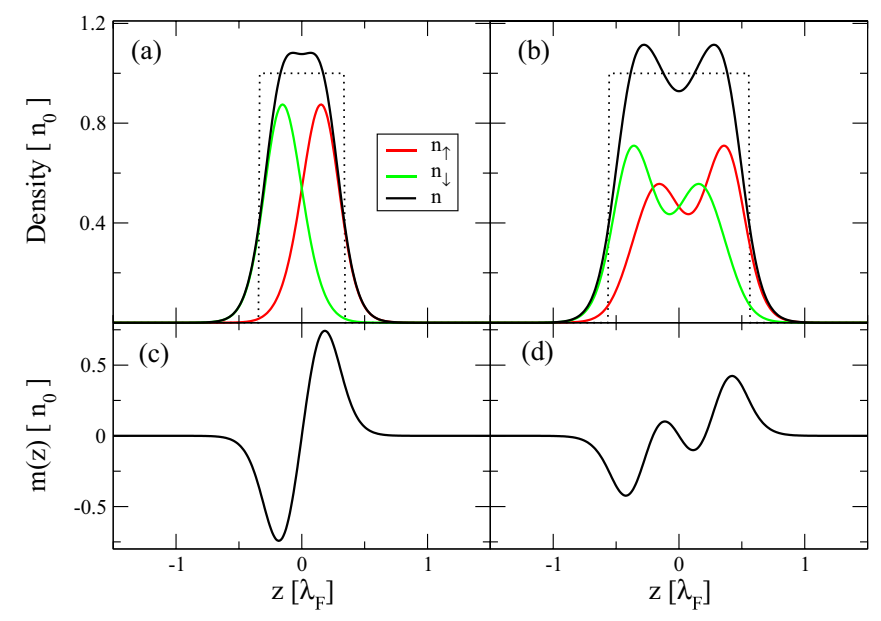

FIG. 15. $x$-KLI spin-density profiles (top panel) and spin polarization density $m(z)$ for $r_{s}=5.0$ and $d / \lambda_{F}=0.68$ (left panel) and $d / \lambda_{F}=1.12$ (right panel). Both left and right panels correspond to AF states, with $\eta=0$.

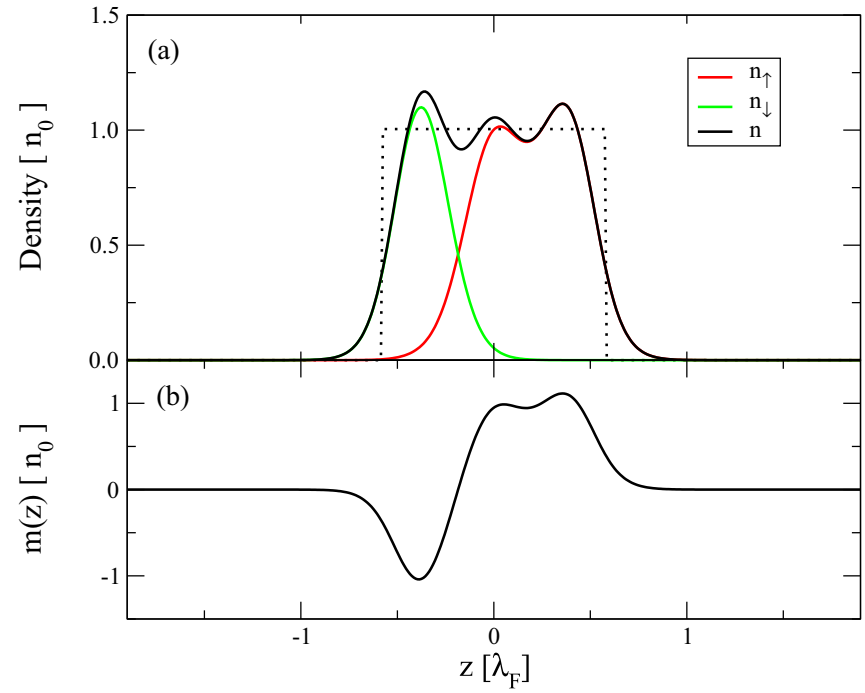

FIG. 16. $x$-KLI spin-density profile (top panel) and spinpolarization density $m(z)$ (lower panel) for a NS-PPF state with $r_{s}=6.0$ and $d / \lambda_{F}=1.16$, and $\eta=0.32$.

profiles for the case $d / \lambda_{F}=0.68$ and $d / \lambda_{F}=1.12$, respectively. Also, in Figs. 15(c) and 15(d), the spin polarization corresponding to the same cases are shown. As can be seen from the figures, a greater overlap between the spin densities is observed in the case of the wider slab. In general, if a greater number of occupied subbands for the AF state occurs, then a greater degree of overlap between the spin densities is observed. For $r_{s}=6$, the AF states are stable in slab widths $0.52 \lesssim d / \lambda_{F}$, showing characteristics similar to those found in the $x$-LSDA results.

The NS-PPF state, that is characterized by $\eta \neq 0$ and its electron density not symmetrical with respect to the center of the slab, is stable in slab widths within the intervals $0.92 \lesssim$ $d / \lambda_{F} \lesssim 1.24,1.32 \lesssim d / \lambda_{F} \lesssim 1.64$ and $1.72 \lesssim d / \lambda_{F} \lesssim 2.00$ for $r_{s}=6$. As it happens in symmetric states, the discontinuities in the curve $\mathcal{H}(d, \eta)$ for $x$-KLI are crucial for the nonoccurrence of stability in some slab widths. Figure 16(a) shows typical spin-density profiles of PPF states for the case of $r_{s}=6.0$ and $d / \lambda_{F}=1.16$, for $\eta=0.32$. Also, in Fig. 16(b), the spin-polarization density corresponding to the same case is shown. As explained in the next section, this configuration is the slab ground state for this particular value of $r_{s}$ and $d$.

The results detailed in this section show a great variety of magnetic structures. By way of summary, Fig. 17 shows all the stable magnetic configurations (symmetric and NS) obtained in the approximation $x$-KLI for the densities and sizes of systems studied.

\section{B. Ground state}

As in the $x$-LSDA case, by comparing the total energy of the different states, the ground state of the system is determined. Figure 18 shows the ground-state configurations which are found in metallic jellium slabs from the exhaustive search in the studied region of densities and magnetizations. It is observed that, as it happens in $x$-LSDA, the ground state is a FPF symmetric state for all the slabs narrower than a specific 


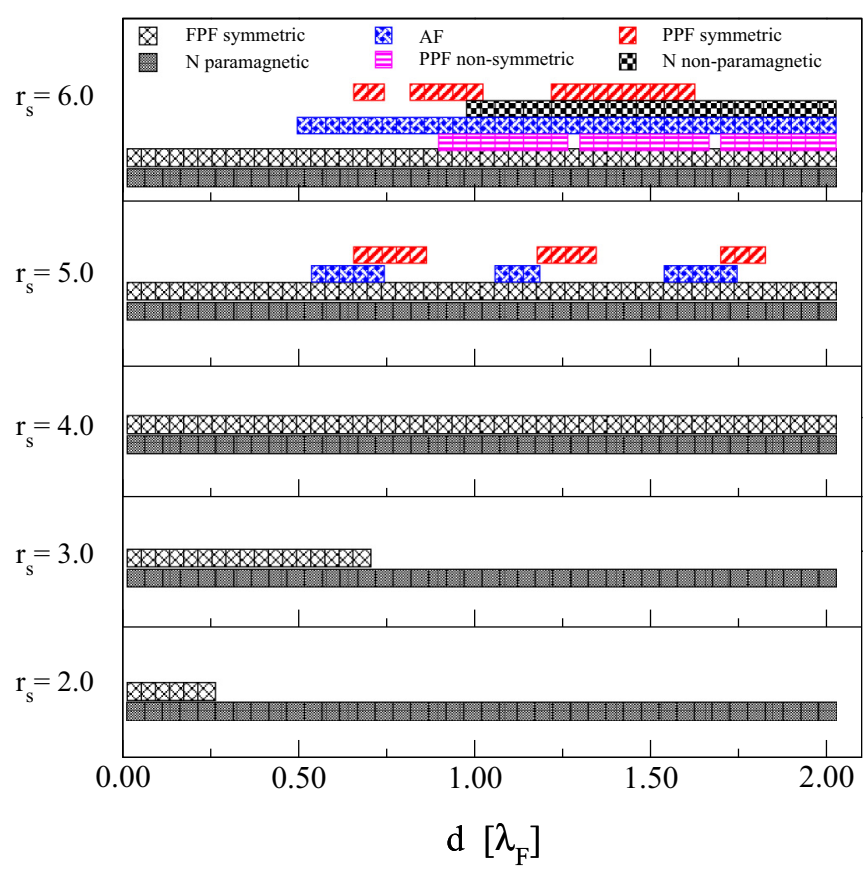

FIG. 17. Jellium slab magnetic structures as resulting from $x$ only total energy KLI calculations in the $r_{s}$ vs $d$ plane.

width that depends on each value of $r_{s}$. For wider slabs, we find different situations for the densities studied. In the cases $r_{s}=2$ and $r_{s}=3$, the ground state is an $\mathrm{N}$ symmetric state, as in $x$-LSDA. The same happens for $r_{s}=4$ and this makes a difference with the $x$-LSDA results because the ground state is never a PPF symmetric state. For $r_{s}=5$, when increasing the width of the slab the fundamental state goes from being a $\mathrm{N}$ symmetric state to an AF state and then a PPF symmetric state. This N-AF-PPF cycle is repeated by increasing the width of the slab even more. Finally, in the case $r_{s}=6$, the ground state is an FPF symmetric state except in one region where it is the $\mathrm{AF}$ state and in another small region where the ground state is NS-PPF.

There is a growing amount of literature devoted to the comparison of the generalized KS hybrids and local or semilocal density approximations [47]; in particular and in connection with the present paper, magnetic ground states have

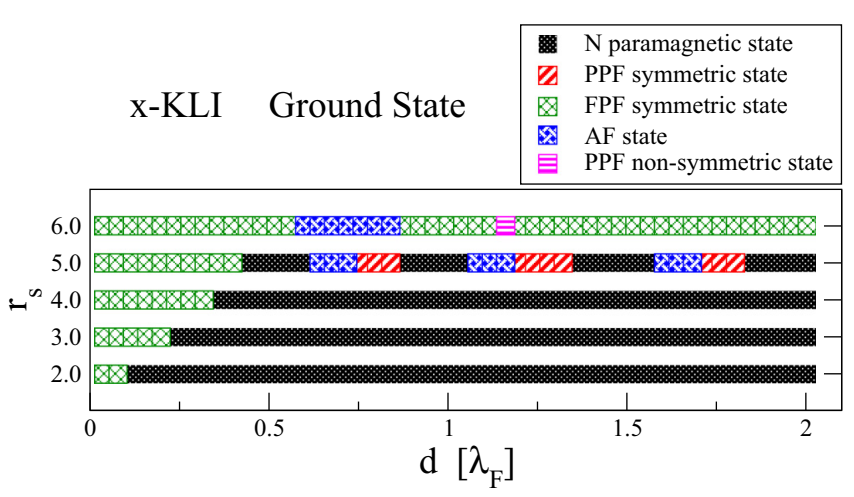

FIG. 18. Jellium slab ground-state configurations as resulting from $x$-only total energy KLI calculations in the $r_{s}$ vs $d$ plane. been predicted for bulk systems $[48,49]$ and surface systems [50]. These hybrid functionals replace a fraction of the local exchange by a nonlocal Hartree-Fock exchange potential. On the other side, two of the authors of the present paper have made, in the past, a direct comparison of the OEP and HF self-consistent calculations for jellium slabs, assuming a paramagnetic situation [51]. It is suggested here that it will be interesting to perform the same comparison for the present narrow slabs, since this may shed some light about the performance of hybrid functionals for slabs.

\section{CONCLUSIONS}

In summary, our systematic study has revealed the different stable magnetic configurations for metallic jellium slabs, using a spin-dependent $x$-only KLI approximation for the exchange potential. This systematic study has been carried out for jellium slabs up to slab widths $d / \lambda_{F}=2$ and $2.0 \leqslant r_{s} \leqslant 6.0$. To explore the different magnetic states present in the metallic jellium slabs, we have resorted to the FSM method.

As a guide for the main objective of this paper, namely, the study of metallic jellium slabs using the KLI approximation, we have first studied these systems using the $x$-LSDA. Depending on the density of the metallic jellium slab and its width, we have found numerous stable magnetic solutions that can be classified into two groups, states with symmetrical spin densities regarding the slab center (symmetric states) or without this symmetry (NS states). Among the states with symmetrical spin densities, we have found the FPF state, the PPF, and $\mathrm{N}$ states. About $\mathrm{N}$ states, we have found the paramagnetic and the nonparamagnetic ones. The last state is characterized by having opposite local relative spin polarization between the center and the edges of the slab. In the case of NS states, we have found the AF state and the NS-PPF state. Finally, we have determined the ground states in the whole region of values of densities and widths of slabs studied. Basically, in the wider slabs the fundamental state is of the FPF symmetric type at low electronic densities $\left(r_{s}=6\right)$ and of the $\mathrm{N}$ paramagnetic type at higher electronic densities $\left(r_{s} \lesssim 4\right)$. For sufficiently narrow slabs, the ground state corresponds to an FPF symmetric state, even for high electronic densities. In the case $r_{s}=5$, it is observed that, depending on the slab width, the ground state can be the FPF symmetric, N paramagnetic, PPF symmetric, or AF state.

In the study of metallic jellium slabs using the $x$-KLI approximation, we have first identified two qualitative differences with the $x$-LSDA study in the FSM procedure to find the different magnetic states. First, the fact that the $x$-KLI potential is not a functional derivative, and second, the presence of abrupt changes in the subband occupancies, that leads to hysteresis in the associated magnetic field curves. These phenomena of hysteresis and discontinuities, absent in an $x$-LSDA study, requires special attention in these regions to determine the possible magnetic states. Basically, we have found that in the regions of hysteresis and discontinuities, no stable states are found. Depending on the density of the metallic jellium slab and its width, we have found all possible stable magnetic solutions presented in $x$-LSDA study. In the case of symmetrical spin-densities, the FPF state, the PPF 
state, the $\mathrm{N}$ paramagnetic state, and the $\mathrm{N}$ nonparamagnetic state are obtained. In the case of NS spin-densities, we have found the AF state and the NS-PPF, as in $x$-LSDA. Finally, we have determined the ground state in the whole region of values of densities and widths of slabs studied. As it happens in $x$-LSDA, the ground-state configuration consists of a FPF symmetric state for all the slabs narrower than a specific width that depends on each value of $r_{s}$. For wider slabs, and $r_{s}=2$ and $r_{s}=3$, the ground state is represented by an $\mathrm{N}$ symmetric state. The same happens for $r_{s}=4$, and this makes a difference with the $x$-LSDA results because the ground state is never a PPF symmetric state. For $r_{s}=5$, we have found, depending of the slab width, as ground state the $\mathrm{N}$ symmetric state, the AF state, and the PPF symmetric state. Finally, in the case $r_{s}=6$, the ground state is conformed by the FPF symmetric state except for some widths where it is represented by the AF state and by the NS-PPF state.

Using the information included in Figs. 9 and 17, the following estimates can be made. Concerning the $x$-LSDA, the ground state is $\mathrm{N}$ paramagnetic in the $63.2 \%$ of the all possible configurations, is FPF symmetric in the $30 \%$, PPF in a $6 \%$, and AF NS in a $0.8 \%$. The same estimates in the $x$-only KLI approximation are as follows: $\mathrm{N}$ paramagnetic $(62.4 \%), \mathrm{FPF}$ symmetric $(26.8 \%)$, AF (6.4\%), PPF symmetric (4\%), and NS-PPF $(0.4 \%)$. Within a general context of similar estimates, we conclude from here that $x$-KLI mainly favors the AF state in comparison with the $x$-LSDA.

The inclusion of correlation effects compatible with the spin-dependent EXX approach discussed here is left for future development. In principle, the same orbital-dependent correlation energy functional as the one used in Refs. $[13,14]$ in the context of semiconductor quantum wells may be also used here, at least for the case of narrow slabs with a few occupied subbands.

\section{ACKNOWLEDGMENTS}

C.M.H was supported by the Consejo Nacional de Investigaciones Científicas y Técnicas (CONICET) of Argentina. C.R.P. thanks CONICET for partial financial support. C.M.H. and C.R.P. acknowledge support from CONICET under Grant No. PIP 2014-2016/00402. C.R.P. acknowledge support from ANPCyT under Grant No. PICT-2016-1087. We thank UnCaFiQT (SNCAD) for computational resources.
[1] R. G. Parr and W. Yang, in Density-Functional Theory of Atoms and Molecules (Oxford University Press, New York, 1989).

[2] R. M. Dreizler and E. K. U. Gross, in Density Functional Theory (Springer, Berlin, 1990).

[3] E. Engel and R. M. Dreizler, Density Functional Theory: An Advanced Course (Springer, Berlin, 2011).

[4] A. E. Mattsson, Science 298, 759 (2002).

[5] J. D. Talman and W. F. Shadwick, Phys. Rev. A 14, 36 (1976).

[6] T. Grabo, T. Kreibich, S. Kurth, and E. K. U. Gross, Strong Coulomb Correlations in Electronic Structure Calculations: Beyond the Local Density Approximation, edited by V. Anisimov, Advances in Condensed Matter Science (Gordon and Breach, Amsterdam, 2000), p. 203.

[7] S. Kümmel and L. Kronik, Rev. Mod. Phys. 80, 3 (2008).

[8] S. Rigamonti, C. M. Horowitz, and C. R. Proetto, Phys. Rev. B 92, 235145 (2015).

[9] C. M. Horowitz, C. R. Proetto, and S. Rigamonti, Phys. Rev. Lett. 97, 026802 (2006).

[10] C. M. Horowitz, C. R. Proetto, and J. M. Pitarke, Phys. Rev. B 78, 085126 (2008).

[11] C. M. Horowitz, L. A. Constantin, C. R. Proetto, and J. M. Pitarke, Phys. Rev. B 80, 235101 (2009).

[12] C. M. Horowitz, C. R. Proetto, and J. M. Pitarke, Phys. Rev. B 81, 121106(R) (2010).

[13] S. Rigamonti and C. R. Proetto, Phys. Rev. B 73, 235319 (2006).

[14] S. Rigamonti and C. R. Proetto, Phys. Rev. Lett. 98, 066806 (2007).

[15] E. Engel, J. Chem. Phys. 140, 18A505 (2014).

[16] E. Engel, Phys. Rev. B 89, 245105 (2014).

[17] E. Engel, Phys. Rev. B 97, 075102 (2018).

[18] E. Engel, Phys. Rev. B 97, 155112 (2018).

[19] E. Engel, Computation 6, 35 (2018).
[20] For metallic paramagnetic jellium slabs, the fact that the asymptotic exact exchange potential decays like $-e^{2} / z$ was proven rigorously in Ref. [9].

[21] A good discussion of the differences between the full OEP and KLI approximations to the exchange potential, within a somehow different context, is given in Phys. Rev. Lett. 93, 213002 (2004).

[22] K. Okazaki and Y. Teraoka, Phys. Rev. B 62, 500 (2000).

[23] P. Sen, Pramana 74, 653 (2010).

[24] N. D. Lang and W. Kohn, Phys. Rev. B 1, 4555 (1970).

[25] N. D. Lang and W. Kohn, Phys. Rev. B 3, 1215 (1971).

[26] They amount to the choice of $\mathrm{H}$ (Hartree) as unit of energy $(\mathrm{H}$ $=27.2 \mathrm{eV})$, and the Bohr radius $a_{0}$ as unit of length $\left(a_{0}=\right.$ $0.529 \AA$ ). An alternative length unit, more useful for the present Q2DEG systems, is $\lambda_{F}=\left(32 \pi^{2} / 9\right)^{1 / 3} r_{s} a_{0} \simeq 3.274 r_{s} a_{0}$. The ratio $d / \lambda_{F}$ characterizes the slab as "narrow" or "wide,", depending on if this ratio is small or large, respectively.

[27] $r_{s}$ being the dimensionless electron-density parameter defined as the radius of a sphere containing one electron on average, and $a_{0}$ being the Bohr radius.

[28] G. Bastard, Wave Mechanics Applied to Semiconductor Heterostructures (Les Editions de Physique, Les Ulis Cedex, France), p. 14.

[29] G. F. Giuliani and G. Vignale, Quantum Theory of the Electron Liquid (Cambridge University Press, Cambridge, 2005), p. 164.

[30] The full factorization in Eq. (2) is, on the other side, not valid in the Hartree-Fock (HF) approximation, since in this case the HF potential is nonlocal. For more details, see Ref. [51]

[31] R. Cortes-Huerto and P. Ballone, Phys. Rev. B 81, 205418 (2010).

[32] P. Cassam-Chenaï, Theor. Chem. Acc. 134, 125 (2015).

[33] J. B. Krieger, Y. Li, and G. J. Iafrate, Phys. Rev. A 45, 101 (1992). 
[34] S. Rigamonti, C. R. Proetto, and F. A. Reboredo, Europhys. Lett. 70, 116 (2005).

[35] Note that we use the symbol $\delta$ to denote a variation with respect to a position-dependent variable, while we use the usual symbol $\partial$ for partial derivatives with respect to a position-independent variable.

[36] The discussion here, in the context of the KLI approximation, follows closely an analogous discussion in Ref. [8], for the full OEP exchange potential. For instance, the limit $V_{x \sigma}^{\mathrm{KLI}}(z \rightarrow$ $\infty) \rightarrow-e^{2} / z\left(1+\beta_{\sigma} / z\right)$, where $\beta_{\sigma}=\bar{z}^{m_{\sigma}}-2 /\left(\pi \sqrt{n_{m_{\sigma} \sigma}}\right)$, is easily obtained from Eq. (11), by considering that for $z / d \gg 1$, the sum is dominated by the last occupied (more energetic) slab energy level. Since the same happens in Eq. (4), the prefactor $\theta_{i \sigma} n_{i \sigma} \xi_{i \sigma}(z \rightarrow \infty)^{2} / n_{\sigma}(z \rightarrow \infty)$ approaches asymptotically the unit value, with $i=m_{\sigma}$. For arriving to the quoted asymptotic result, it only remains to fix the unique floating constant implicit in Eq. (11) by imposing the condition $\overline{\Delta V}_{x m_{\sigma \sigma}}=0$, and consider that $u_{x m_{\sigma} \sigma}(z \rightarrow \infty) \rightarrow-e^{2} / z\left(1+\beta_{\sigma} / z\right)$ [9].

[37] W. Kohn and A. E. Mattsson, Phys. Rev. Lett. 81, 3487 (1998).

[38] M. Abramowitz and I. A. Stegun, Handbook of Mathematical Functions (Dover, New York, 1964).

[39] K. Schwarz and P. Mohn, J. Phys. F 14, L129 (1984).

[40] A. R. Williams, V. L. Moruzzi, J. Kübler, and K. Schwarz, Bull. Am. Phys. Soc. 29, 278 (1984).
[41] P. H. Dederichs, S. Blügel, R. Zeller, and H. Akai, Phys. Rev. Lett. 53, 2512 (1984).

[42] U. von Barth and L. Hedin, J. Phys. C 5, 1629 (1972).

[43] J. F. Janak, V. L. Moruzzi, and A. R. Williams, Phys. Rev. B 12, 1257 (1975).

[44] The situation at $\eta$ around 1 is such that $\mathcal{H}(\eta \rightarrow 1)$ and $d \mathcal{H}(\eta) /\left.d \eta\right|_{\eta \rightarrow 1}$ become large and negative as we get closer to $\eta=1$. This behavior is a consequence of the greater tendency of the system to go to the FPF state and therefore the need to counteract this trend with larger values of $\mathcal{H}$, as we approach $\eta=1$. Furthermore, at $\eta=1, \mathcal{H}(\eta=1)=0$. In other words, the function $\mathcal{H}(\eta)$ is discontinuous at $\eta=1$, and its derivative is then not defined.

[45] F. Bloch, Z. Phys. 57, 545 (1929).

[46] A. Karolewski, R. Armiento, and S. Kümmel, Phys. Rev. A 88, 052519 (2013).

[47] J. P. Perdew, W. Yang, K. Burke, Z. Yang, E. K. U. Gross, M. Scheffler, G. E. Scuseria, T. M. Henderson, I. Y. Zhang, A. Ruzsinszky et al., Proc. Natl. Acad. Sci. USA 114, 2801 (2017).

[48] V. Eyert, Phys. Rev. Lett. 107, 016401 (2011).

[49] S. Xu, X. Shen, K. A. Hallman, R. F. Haglund, Jr., and S. T. Pantelides, Phys. Rev. B 95, 125105 (2017).

[50] B. Pamuk and M. Calandra, Phys. Rev. B 99, 155303 (2019).

[51] H. Luo, C. M. Horowitz, H.-J. Flad, C. R. Proetto, and W. Hackbusch, Phys. Rev. B 85, 165133 (2012). 